\title{
A Learning Based Statistical Approach for Combining Multiple Measurements in Electrocardiographic Imaging
}

\author{
Taha Erenler ${ }^{1,2}$, Yeşim Serinağaoğlu Doğrusöz ${ }^{2}$ \\ ${ }^{1}$ HAVELSAN Inc. , Ankara, Turkey \\ ${ }^{2}$ Electrical and Electronics Engineering Department, Middle East Technical University, Turkey
}

\begin{abstract}
Kalman filter has been applied in literature to inverse electrocardiography problem as a spatio-temporal method. However, there is still an open question of how the essential parameters in the state-space representation are found without claiming strong assumptions. In this study, we proposed a maximum likelihood (ML) estimation based method which incorporates multiple body surface measurements to estimate the parameters that are essential to use Kalman filter.

Our proposed approach, Maximum Likelihood Inference \& Filtering (MLIF), was compared with zero order Tikhonov regularization and Bayesian maximum a posteriori estimation (BMAP) by using three different training sets under two different measurement noise levels. The results showed that mean correlation coefficient (CC) for Tikhonov regularization is 0.60 , and mean CC ranges 0.64 to 0.82, and 0.66 to 0.99 for Bayesian MAP and MLIF under $30 \mathrm{~dB}$ SNR measurement noise, respectively. Under $10 \mathrm{~dB} S N R$, mean CC is 0.37 for Tikhonov regularization, and mean CC ranges 0.53 to 0.78 , and 0.53 to 0.98 for Bayesian MAP and MLIF, respectively.
\end{abstract}

\section{Introduction}

Cardiac diseases are among the leading causes of death in the world. Early diagnosis is very important to prevent these deaths like any other diseases. 12-lead electrocardiography (ECG) is the mostly used technique for the diagnosis of cardiovascular diseases. 12-lead ECG is a very efficient, quick and patient-friendly method but it suffers from spatial smoothing of cardiac signals within the torso, and low spatial resolution, and some abnormalities in the heart function cannot be detected by clinicians using this method. Electrocardiographic imaging (ECGI), also known as inverse electrocardiography (ECG), aims to determine the cardiac electrical activity from body surface potential measurements. Reconstruction of the cardiac electrical activity from the body surface potential measure- ments is not an easy task, since this problem has an illposed nature due to attenuation and spatial smoothing inside the medium between the source and the measurement sites, meaning that even small errors in the mathematical model or noise in the measurements may yield unbounded errors or large oscillations in the solutions [1]. One remedy for this ill-posedness is to apply regularization, where one imposes deterministic or statistical constraints on the solution based on available a priori information.

Tikhonov regularization, Bayesian maximum a posteriori estimation [2] and Kalman filter have been applied to inverse ECG problem in this study. In the context of Kalman filter, the researchers focused on how Kalman filter's parameters should be found. For example, Joly et al. [3] attempted to model the state transition matrix as a constant times times identity matrix and this approach is a poor approximation since it ignores the relation between the state variables. Based on Joly et al.'s study, El-Jakl et al. [4] proposed another method to find the state transition matrix by using ML estimation. However, El-Jakl's study assumed the covariance matrix to be a constant times identity matrix and did not provide information on how the multiple measurements were used. More recently, Aydin et al. [5] assumed epicardial potential at one lead is related only to the leads in its neighbourhood or the leads that are activated at around the same time, while finding the state transition matrix. Hence, in their study, it is important how the neighbourhood leads are defined. Furthermore, any errors that are made during the calculation of activation times may yield incorrect state transition matrix.

In this study, we propose a new method which incorporates multiple measurements by using ML estimation. Our approach provides a general way to construct the state-space formulation of the inverse electrocardiography by using very few assumptions. After the construction of the statespace formulation of the problem, we apply Kalman filter and smoother to the inverse ECG problem. 


\section{Maximum Likelihood Inference \& Fil- tering (MLIF)}

The relation between the heart sources and the body surface measurements becomes linear when epicardial potentials are used as cardiac source [6]. This relation can be represented as follows in state-space form:

$$
\begin{aligned}
y_{k} & =H x_{k}+v_{k} \\
x_{k+1} & =F x_{k}+w_{k}
\end{aligned}
$$

where

- $x_{k} \in \mathbb{R}^{\mathbb{M}}$ denotes $M$ dimensional epicardial state vector at time $k$,

- $y_{k} \in \mathbb{R}^{\mathbb{D}}$ denotes $D$ dimensional body surface potential vector at time $k$,

- $F \in \mathbb{R}^{\mathbb{M x M}}$ is the state transition matrix,

- $H \in \mathbb{R}^{\mathbb{D x M}}$ is the forward transfer matrix,

- $w_{k} \sim \mathcal{N}\left(w_{k} ; 0, Q\right)$ is the Gaussian process noise with zero mean and covariance matrix $Q^{M \times M}$, assumed to be uncorrelated with the state,

- $v_{k} \sim \mathcal{N}\left(v_{k} ; 0, R\right)$ is the Gaussian process noise with zero mean and covariance matrix $R^{D \times M}$, assumed to be uncorrelated with the state,

- $x_{1} \sim \mathcal{N}(\bar{x}, \Sigma)$ is the initial epicardial potential state.

Kalman filter and smoother is applied to system (1) in the

MLIF method. Discrete Kalman filter steps are as follows:

Step 1. Initialization

For $\mathrm{k}=1$, initialize $x_{k}$ and $P_{k}$

- $\hat{x}_{1 \mid 1}=\mathbf{E}\left[x_{1}\right]=\bar{x}$

- $P_{1 \mid 1}=\mathbf{E}\left[\left(x_{1}-\hat{x}_{1 \mid 1}\right)\left(x_{1}-\hat{x}_{1 \mid 1}\right)^{T}\right]=\Sigma$

Step 2. Forward Recursion: Filtering

For $\mathrm{k}=2, \ldots, \mathrm{T}$ do

- $\hat{x}_{k \mid k-1}=F \hat{x}_{k-1 \mid k-1}$

- $P_{k \mid k-1}=F P_{k-1 \mid k-1} F^{T}+Q$

- $K_{k}=P_{k \mid k-1} H^{T}\left(H P_{k \mid k-1} H^{T}+R\right)^{-1}$

- $\hat{x}_{k \mid k}=\hat{x}_{k \mid k-1}+K_{k}\left(y_{k}-H \hat{x}_{k \mid k-1}\right)$

- $P_{k \mid k}=\left(I-K_{k} H\right) P_{k \mid k-1}$

After the Kalman filter steps, our next step is the Kalman smoother. In this study, we use Rauch-Tung-Striebel Smoother (RTS) [7]. RTS is a very convenient approach for linear Gaussian models, as in our model. RTS steps are given as follows:

Step 3. Backward Recursion: Smoothing

For $\mathrm{k}=\mathrm{T}-1, \ldots ., 1$ do

- $P_{k+1 \mid k}=F P_{k \mid k} F^{T}+Q$

- $G_{k}=P_{k \mid k} F^{T}\left(P_{k+1 \mid k}\right)^{-1}$

- $\hat{x}_{k \mid T}=\hat{x}_{k \mid k}+G_{k}\left[\hat{x}_{k+1 \mid T}-F \hat{x}_{k \mid k}\right]$

In Kalman filter and smoother steps, $F, Q, R$ and, the initial state's mean and covariance $\bar{x}, \Sigma$ are needed. These parameters are found by using by using ML parameter estimation technique.

\section{Maximum Likelihood (ML) Parame- ter Estimation}

Parameter estimation based on ML method was used extensively in speech signal processing literature [8,9]. ML estimation finds the best estimate of the unknown parameter set $\Theta=\{\bar{x}, \Sigma, F, Q, R\}$, which maximizes the joint likelihood function, so that the observed data is the most probable.

We start by selecting L experiments from an epicardial potential database and simulate body surface potentials for each experiment. Then, we compose a training set with epicardial potentials $\mathrm{X}=\left\{x_{1: T}^{\ell}\right\}_{\ell=1}^{L}$ and simulated body surface potentials $\mathrm{Y}=\left\{y_{1: T}^{\ell}\right\}_{\ell=1}^{L}$. Mathematically, we find the value of the parameter set as follows:

$$
\hat{\Theta}=\underset{\Theta}{\operatorname{argmax}} \underbrace{p(X, Y \mid \Theta)}_{\Pi(\Theta)}
$$

where

$$
\Pi(\Theta)=\prod_{\ell=1}^{L} p\left(x_{1: T}^{\ell}, y_{1: T}^{\ell} \mid \Theta\right)
$$

under the assumption of independent experiments. Assuming Markov property, $\Pi(\Theta)$ is written as follows:

$\Pi(\Theta)=\prod_{\ell=1}^{L}\left(p\left(x_{1}^{\ell} \mid \Theta\right) \prod_{k=1}^{T} p\left(y_{k}^{\ell} \mid x_{k}^{\ell}, \Theta\right) \prod_{k=2}^{T} p\left(x_{k}^{\ell} \mid x_{k-1}^{\ell}, \Theta\right)\right)$

We take the natural logarithm of $\Pi(\Theta)$ and plug the parameter set into equation (4):

$$
\begin{aligned}
\ln (\Pi(\Theta)) & =\sum_{\ell=1}^{L} \ln p\left(x_{1}^{\ell} \mid \bar{x}, \Sigma\right)+\sum_{\ell=1}^{L} \sum_{k=1}^{T} \ln p\left(y_{k}^{\ell} \mid x_{k}^{\ell}, R\right) \\
& +\sum_{\ell=1}^{L} \sum_{k=2}^{T} \ln p\left(x_{k}^{\ell} \mid x_{k-1}^{\ell}, F, Q\right)
\end{aligned}
$$

where

- $p\left(x_{1}^{\ell} \mid \bar{x}, \Sigma\right) \sim \mathcal{N}\left(x_{1}^{\ell} ; \bar{x}, \Sigma\right)$,

- $p\left(y_{k}^{\ell} \mid x_{k}^{\ell}, R\right) \sim \mathcal{N}\left(y_{k}^{\ell} ; H x_{k}^{\ell}, R\right)$,

- $p\left(x_{k}^{\ell} \mid x_{k-1}^{\ell}, F, Q\right) \sim \mathcal{N}\left(x_{k}^{\ell} ; F x_{k-1}^{\ell}, Q\right)$.

To find the parameter set $\{\bar{x}, \Sigma, F, Q, R\}$, we take the derivative of $\ln (\Pi(\Theta))$ with respect to each component and obtain the roots by equating it to zero.

We summarize the resulting estimates in Table 1. 
Table 1. ML based parameter estimation

\begin{tabular}{l}
\hline \hline Estimated parameters: \\
$\hat{\bar{x}}=\frac{1}{L} \sum_{\ell=1}^{L} x_{1}^{\ell}$ \\
$\hat{\Sigma}=\frac{1}{L} \sum_{\ell=1}^{L}\left(x_{1}^{\ell}-\bar{x}\right)\left(x_{1}^{\ell}-\bar{x}\right)^{T}$ \\
$\hat{R}=\frac{1}{L T} \sum_{\ell=1}^{L} \sum_{k=1}^{T}\left(y_{k}^{\ell}-H x_{k}^{\ell}\right)\left(y_{k}^{\ell}-H x_{k}^{\ell}\right)^{T}$ \\
$\hat{F}=\left[\sum_{\ell=1}^{L} \sum_{k=2}^{T} x_{k}^{\ell}\left(x_{k-1}^{\ell}\right)^{T}\right]$ \\
$\hat{Q}=\frac{1}{L(T-1)} \sum_{\ell=1}^{L} \sum_{k=2}^{T}\left(x_{k}^{\ell}-F x_{k-1}^{\ell}\right)\left(x_{k}^{\ell}-F x_{k-1}^{\ell}\right)^{T}$ \\
\hline \hline
\end{tabular}

\section{Bayesian Maximum a Posteriori Esti- mation (BMAP)}

Bayes' theorem states that posterior pdf of $x$ given $y$ can be found by using the following equation [2]:

$$
\underbrace{p(x \mid y)}_{\text {Posterior pdf }}=\frac{\overbrace{p(y \mid x)}^{\text {Likelihood Prior pdf }} \overbrace{p(x)}^{\int_{p(y \mid x) P(x) d x}}}{\underbrace{}_{\text {Evidence }} p(y \mid x)}
$$

Bayesian MAP estimation technique maximizes the posterior pdf of $x$ given $y$. By maximizing the posterior pdf, we find the most probable value of $x$ given observed data $y$. Maximization of the posterior pdf is given by the following equation:

$$
\hat{x}=\underset{x}{\operatorname{argmax}} p(x \mid y)=\underset{x}{\operatorname{argmax}} \frac{p(y \mid x) p(x)}{p(y)}
$$

We assume that:

- $p(y \mid x) \triangleq \mathcal{N}(y ; H x, R)$

- $p(x) \triangleq \mathcal{N}(x ; \bar{x}, \Sigma)$

where $\bar{x}=E\left[x_{k}\right]$ and $\Sigma=E\left[\left(x_{k}-\bar{x}_{k}\right)\left(x_{k}-\bar{x}_{k}\right)^{T}\right] \forall k$.

To maximize equation (7), we take the derivative of the $\log$ of that equation and find the root by setting the derivative equal to zero. After doing the necessary calculations, $\hat{x}$ is found as follows:

$$
\hat{x}=\left(H^{T} R^{-1} H+\Sigma^{-1}\right)^{-1}\left(H^{T} R^{-1} y+\Sigma^{-1} \bar{x}\right)
$$

The training set in BMAP is composed by following Serinagaoglu et al.'s approach [2].

\section{Results and Conclusion}

In this study, three different training sets are used. The training sets are were constructed as follows:

- Scenario 1: In scenario 1, the beat we used for simulating the measurements (i.e., the test beat) and the training set beats came from the same experiment (same dog heart). The test beat was included in the training set.

- Scenario 2: Leave-One-Beat-Out Protocol [2]: In scenario 2, we used the same training set as in scenario 1 , but excluded the test beat.

- Scenario 3: Leave-One-Experiment-Out Protocol [2]: In scenario 3 , the test beat came from a different experiment (i.e., a different dog heart) than the experiments from which we obtained the training set beats.

In this study, University of Utah's epicardial potential database was used. Body surface potentials were calculated by simulation, i.e., the epicardial potentials were multiplied by the forward transfer matrix, which was found by using boundary element method, and a proper noise (10 $\mathrm{dB}$ or $30 \mathrm{~dB}$ SNR) was added to the result.

For all the scenarios, 100 Monte Carlo runs were used to obtain the results. The average of all these 100 simulations was taken in each case.

In order to quantitatively compare performances of the proposed algorithms, correlation coefficient (CC) between the real and the reconstructed epicardial potential distributions is used. The correlation coefficient can be defined as follows:

$C C_{x, \hat{x}}(k)=\frac{\sum_{i=1}^{M}\left(\hat{x}_{i}(:, k)-\overline{\hat{\mathbf{x}}}(:, k)\right)\left(x_{i}(:, k)-\overline{\mathbf{x}}(:, k)\right)}{\|\hat{\mathbf{x}}(:, k)-\overline{\hat{\mathbf{x}}}(:, k)\| \cdot\|\mathbf{x}(:, k)-\overline{\mathbf{x}}(:, k)\|}$

where $\|\cdot\|$ is the L2 norm, $M$ is the number of epicardial nodes, $\hat{\mathbf{x}}_{k}$ is the estimated epicardial potential state vector and $\mathbf{x}_{k}$ is real the epicardial potential state vector at time $\mathrm{k}$.

As seen in Tables 2 and 3, MLIF outperforms Tikhonov and BMAP methods at both measurement noise levels. MLIF is more robust to measurement noise since it uses not only spatial correlations but also temporal correlations between epicardial potentials. Due to over-fitting nature of ML estimation, when the training set data and the test beat are not similar, its performance is significantly degraded. This fact can be observed by looking at training set 3 results. Tikhonov regularization is applied independent of the training sets, hence the results are the same in different training sets. Tikhonov regularization is the most affected method among the proposed methods when the SNR is increased from $30 \mathrm{~dB}$ to $10 \mathrm{~dB}$. BMAP uses the training set data as a prior information, hence it is more robust to measurement noise than Tikhonov regularization. 
Table 2. Mean correlation coefficients under $10 \mathrm{~dB}$ measurement noise

\begin{tabular}{lccr}
\hline \hline Method & Training Set 1 & Training Set 2 & Training Set 3 \\
\hline Tikhonov & $0.37 \pm 0.31$ & $0.37 \pm 0.31$ & $0.37 \pm 0.31$ \\
BMAP & $0.78 \pm 0.27$ & $0.77 \pm 0.26$ & $0.53 \pm 0.29$ \\
MLIF & $0.98 \pm 0.02$ & $0.88 \pm 0.11$ & $0.53 \pm 0.30$ \\
\hline \hline
\end{tabular}

Table 3. Mean correlation coefficients under $30 \mathrm{~dB}$ measurement noise

\begin{tabular}{lccr}
\hline \hline Method & Training Set 1 & Training Set 2 & Training Set 3 \\
\hline Tikhonov & $0.60 \pm 0.28$ & $0.60 \pm 0.28$ & $0.60 \pm 0.28$ \\
BMAP & $0.82 \pm 0.18$ & $0.79 \pm 0.19$ & $0.64 \pm 0.26$ \\
MLIF & $0.99 \pm 0.02$ & $0.88 \pm 0.10$ & $0.66 \pm 0.23$ \\
\hline \hline
\end{tabular}

\section{Discussions and Future Works}

In general, spatio-temporal method MLIF outperformed spatial methods and enhanced the reconstruction accuracy. When the training set data and the test beat are similar, finding Kalman filter's parameters with ML estimation yielded better results. However, due to over-fitting, if the training set data and the test beat are not similar, i.e., if they come from different physiological models, the performance of ML estimation was degraded. In such situations, maximum a posteriori estimation can be used to overcome the over-fitting issue [10].

As a future work, maximum a posteriori parameter estimation technique will be implemented.

\section{Acknowledgements}

The authors would like to thank Professor Robert S. MacLeod from University of Utah for the data used in this study.

This work had been partially supported by Havelsan Inc.

\section{References}

[1] Macfarlane, P. W. (2011). The inverse problem of electrocardiography. Comprehensive Electrocardiology, 33(2), 1792.

[2] Serinagaoglu, Y., Brooks, D. H., MacLeod, R. S. (2005). Bayesian solutions and performance analysis in bioelectric inverse problems. IEEE Transactions on Biomedical Engineering, 52(6), 1009-1020.

[3] Joly, D., Goussard, Y., Savard, P. (1993). Time-recursive solution to the inverse problem of electrocardiography: a model-based approach. Proceedings of the 15th Annual International Conference of the IEEE Engineering in Medicine and Biology Society, 1-10.

[4] El-Jakl, J., Champagnat, F., Goussard, Y. (1995). Timespace regularization of the inverse problem of electrocar-

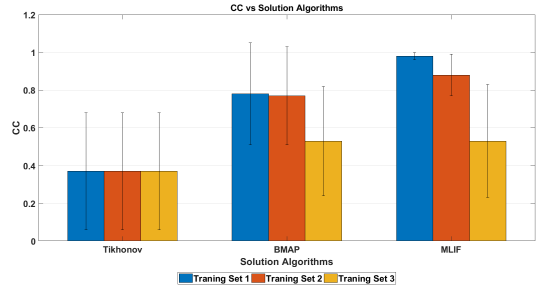

Figure 1. Bar chart representation of Table 2

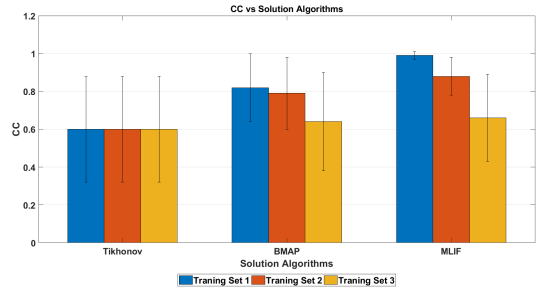

Figure 2. Bar chart representation of Table 3

diography. Proceedings of 17th International Conference of the Engineering in Medicine and Biology Society, 1, 213-214.

[5] Aydin, U., Serinagaoglu, Y. (2009). Use of Activation Time Based Kalman Filtering in Inverse Problem of Electrocardiography., 4th European Conference of the International Federation for Medical and Biological Engineering (pp. 1200-1203).

[6] Yamashita, Y. (1982). Theoretical studies on the inverse problem in electrocardiography and the uniqueness of the solution. IEEE Transactions on Biomedical Engineering, BME-29(11), 719-725.

[7] Rauch, H. E., Striebel, C. T., Tung, F. (1965). Maximum likelihood estimates of linear dynamic systems. AIAA Journal, 3(8), 1445-1450.

[8] Yücel Özbek, I., Demirekler, M. (2010). ML vs. MAP parameter estimation of linear dynamic systems for acousticto-articulatory inversion: A comparative study. European Signal Processing Conference, 805-809.

[9] Katsamanis, A., Ananthakrishnan, G., Papandreou, G., Maragos, P., Engwall, O. (2008). Audiovisual speech inversion by switching dynamical modeling governed by a Hidden Markov process. European Signal Processing Conference, 2-6.

[10] Bishop, C. M. (2006). Pattern Recognition and Machine Learning (Information Science and Statistics).

Address for correspondence:

Assoc. Prof. Dr. Yeşim Serinağaoğlu Doğrusöz

Electrical and Electronics Engineering Department, Middle East Technical University

yserin@metu.edu.tr 